\title{
The mass composition of cosmic rays measured with LOFAR
}

\author{
Jörg R. Hörandel ${ }^{1,2, a}$, A. Bonardi ${ }^{1}$, S. Buitink ${ }^{1,3}$, A. Corstanje ${ }^{1}$, H. Falcke ${ }^{1,2,4,5}$, P. Mitra ${ }^{3}$, K. \\ Mulrey $^{3}$, A. Nelles ${ }^{1,2,8}$, J.P. Rachen ${ }^{1}$, L. Rossetto ${ }^{1}$, P. Schellart ${ }^{1,9}$, O. Scholten ${ }^{6,7}$, S. ter Veen ${ }^{4}$, S. \\ Thoudam $^{1,10}$, T.N.G. Trinh ${ }^{6}$, and T. Winchen ${ }^{3}$ \\ ${ }^{1}$ Department of Astrophysics, IMAPP, Radboud University Nijmegen P.O. Box 9010, 6500 GL Nijmegen, The \\ Netherlands \\ ${ }^{2}$ NIKHEF, Science Park Amsterdam, Amsterdam 1098 XG, The Netherlands \\ ${ }^{3}$ Astrophysical Institute, Vrije Universiteit Brussel, Pleinlaan 2, Brussels 1050, Belgium \\ ${ }^{4}$ ASTRON, Postbus 2, Dwingeloo 7990 AA, The Netherlands \\ ${ }^{5}$ Max-Planck-Institut für Radio Astronomie, Bonn, Germany \\ ${ }^{6}$ KVI-CART, Groningen University, P.O. Box 72, Groningen 9700 AB, The Netherlands \\ ${ }^{7}$ Interuniversity Institute for High Energies, Vrije Universiteit Brussel, Pleinlaan 2, 1050 Brussels, Belgium \\ ${ }^{8}$ now at: Department of Physics and Astronomy, University of California Irvine, Irvine, CA 92697-4575, USA \\ ${ }^{9}$ now at: Department of Astrophysical Sciences, Princeton University, Princeton, NJ 08544, USA \\ ${ }^{10}$ now at: Department of Physics and Electrical Engineering Linneuniversitetet, 35195 Växjö, Sweden
}

\begin{abstract}
High-energy cosmic rays, impinging on the atmosphere of the Earth initiate cascades of secondary particles, the extensive air showers. The electrons and positrons in the air shower emit electromagnetic radiation. This emission is detected with the LOFAR radio telescope in the frequency range from 30 to $240 \mathrm{MHz}$. The data are used to determine the properties of the incoming cosmic rays. The radio technique is now routinely used to measure the arrival direction, the energy, and the particle type (atomic mass) of cosmic rays in the energy range from $10^{17}$ to $10^{18} \mathrm{eV}$. This energy region is of particular astrophysical interest, since in this regime a transition from a Galactic to an extra-galactic origin of cosmic rays is expected. For illustration, the LOFAR results are used to set constraints on models to describe the origin of high-energy cosmic rays.
\end{abstract}

\section{Introduction}

Cosmic rays (ionized atomic nuclei) impinge on the Earth with (kinetic) energies covering a wide range from $\mathrm{MeV}$ energies up to beyond $10^{20} \mathrm{eV}$. At energies below $\sim 100 \mathrm{MeV}$ they are accelerated in energetic outbursts of the Sun. At higher energies, the are assumed to originate in our Milky Way, being accelerated in Supernova remnants (e.g. [1, 2]. At energies exceeding $10^{18} \mathrm{eV}$ it becomes increasinlgy difficult to magnetically bind the particles to our Galaxy. Thus, particles with energies above $\sim 10^{18} \mathrm{eV}$ are usually considered to be of extra-galactic origin. A transition from a Galactic to an extra-galactic origin of cosmic rays is expected at energies around $10^{17}$ to $10^{18} \mathrm{eV}[3,4]$.

In this paper we will shed new light on the understanding of the origin of cosmic rays in the transition region $\left(10^{17}-10^{18} \mathrm{eV}\right)$. This necessitates a precise measurement of the properties of cosmic

\footnotetext{
ae-mail: j.horandel@astro.ru.nl-http://particle.astro.ru.nl
} 
rays, namely their arrival direction (on the sky), their (kinetic) energy, and their particle type (atomic mass $A$ ).

The flux of cosmic rays is steeply falling, approximately following a power law $\propto E^{-3}$. In our region of interest, cosmic rays are only measured indirectly, using large ground-based detector installations. High-energy cosmic rays impinging on the atmosphere, initiate cascades of secondary particles, the extensive air showers. The challenge of the indirect measurements is to derive the properties of the incoming cosmic rays from air-shower observations. Most challenging is the measurement of the particle type, since the sensitivity of air shower measurements is only proportional to $\ln A$. Intrinsic shower fluctuations allow to divide the measured cosmic rays in up to five mass groups for the best experiments [5].

The radio measurement of air showers to determine the properties of cosmic rays is briefly sketched in Sect. 2. The method is used to determine the properties of cosmic rays as outlined in Sect.3. One of the key results obtained is the mass composition of cosmic rays in the transition region. Implications on our understanding of the origin of cosmic arys will be discussed.

\section{Radio detection of air showers}

Many secondary particles in extensive air showers are electrons and positrons. They emit radiation with frequencies of tens of $\mathrm{MHz}$ mainly due to interaction with the magnetic field of the Earth. Radio detection of air showers is suitable to measure the properties of cosmic rays with nearly $100 \%$ duty cycle $[6,7]$.

The LOFAR radio telescope $[8,9]$ is one of the leading installations for the LOFAR radio measurements of air showers. LOFAR is a digital radio telescope. Its antennas are spread over several European countries and are used together for interferometric radio observations in the frequency range of $30-240 \mathrm{MHz}$. The density of antennas increases towards the center of LOFAR, which is located in the Netherlands. Here, about 2400 antennas are clustered on an area of roughly $10 \mathrm{~km}^{2}$ with increasing antenna density towards the center. This high density of antennas makes LOFAR the perfect tool to study features of the radio emission created by extensive air showers. The radio antennas have been calibrated with in-situ measurements, using a reference source and Galactic emission [10].

Air shower measurements are conducted based on a trigger received from an array of scintillators (LORA) [11, 12], which results in a read-out of the ring buffers that store the raw voltage traces per antenna for up to $5 \mathrm{~s}$. LOFAR comprises two types of antennas. While air showers have also been measured in the high-band $(110-240 \mathrm{MHz})$, most air showers are measured with the low-band antennas (LBA), which cover the frequency range from $10-90 \mathrm{MHz}$. The LBAs are arranged in compact clusters of 96 antennas, called stations. Of every station either the inner group or the outer ring of antennas (48 antennas each) can be used for cosmic-ray measurements at a given time.

In the last years the radio technique has been established as a precise method to measure the mass composition of cosmic rays. The LOFAR measurements together with the predictions of the CoREAS [13] simulation package result in a complete understanding of the emission mechanisms. With LOFAR the properties of the radio emission have been measured with high accuracy [14-16] in the frequency range $30-80 \mathrm{MHz}$, which allows us to establish key features, such as the lateral density distribution of the radio signals [17, 18], the shape of the shower front [19], and the polarization of the radio signal $[20,21]$. These measurements help to understand the emission processes in the atmosphere and to quantify the contributions of the two mechanisms, being responsible for the radio emission of air showers - namely the geomagnetic effect (i.e. charge separation in the geomagnetic field) and the Askaryan effect (charge excess in the shower front). We obtained the first quantitative measurements in the frequency range $120-240 \mathrm{MHz}$ [22]. We also recorded air showers during thunderstorm conditions $[23,24]$ and measured the structure of electric fields in the atmosphere. 


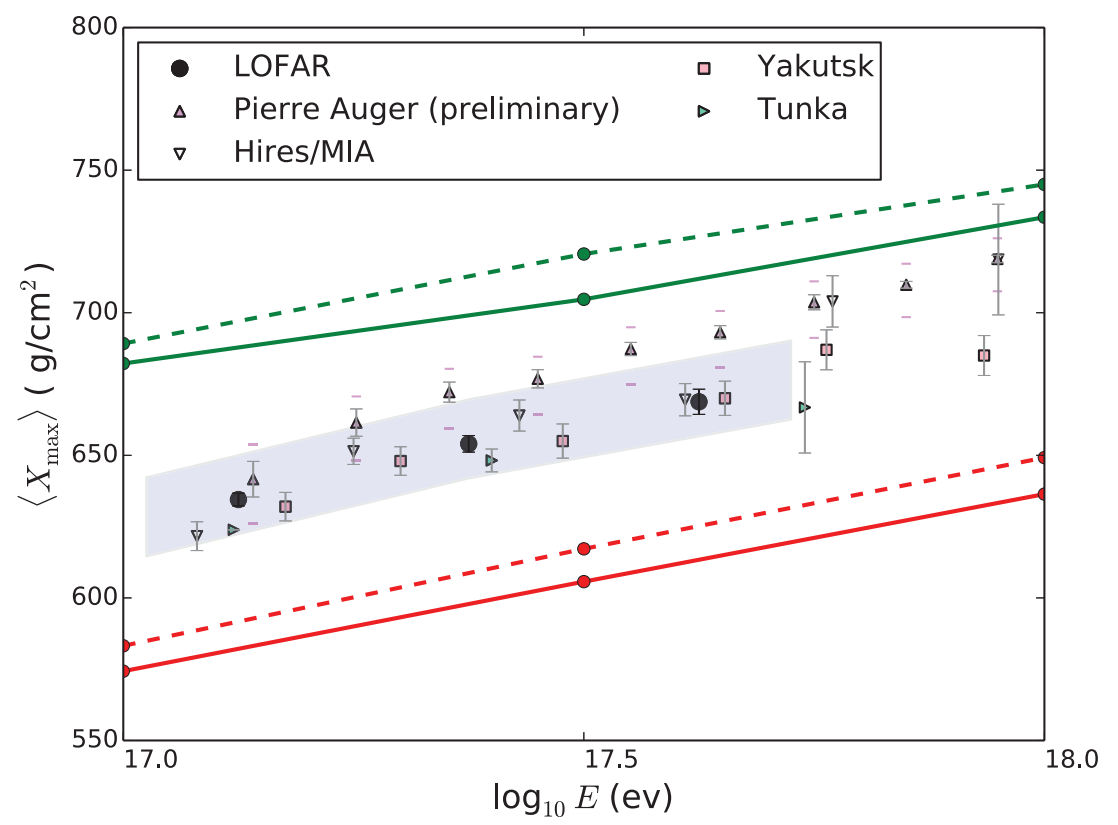

Figure 1. Average depth of the shwoer maximum $X_{\max }$ as a function of cosmic-ray energy [29]. The LOFAR radio results are compared to optical measurements. For details and references see [29]. The lines represent predictions for protons (green) and iron nuclei (red) for the hadronic interaction models QGSJETII.04 (solid) and EPOS-LHC (dashed).

\section{Properties of cosmic rays}

\section{Direction}

The excellent time resolution of LOFAR with ns accuracy allows to measure the shape of the shower front [19]. It is found that a hyperboloid describes the measurements best. Such a behavior has been found earlier by the LOPES experiment [25]. In order to estimate the accuracy of the measurement of the arrival direction of the shower, the same measured air showers have been reconstructed with different assumptions (plane, sphere, hyperboloid). These investigations indicate an uncertainty for the direction reconstruction of the order of $0.1^{\circ}$ to $0.5^{\circ}$.

\section{Energy}

The recording of radio signals in the frequency range of interest $(30-80 \mathrm{MHz})$ provides an excellent calorimetric measure of the energy contained in the electromagnetic component of the air shower and, thus, provides a good measure of the energy of the shower-inducing particle. The integral over the measured energy fluence distribution on the ground is proportional to the energy of the incoming cosmic ray. A resolution around $30 \%$ for the cosmic-ray energy is obtained with LOFAR [26]. Similar investigations at the Pierre Auger Observatory indicate that a resolution around 25\% is possible for high-quality showers [27, 28]. 

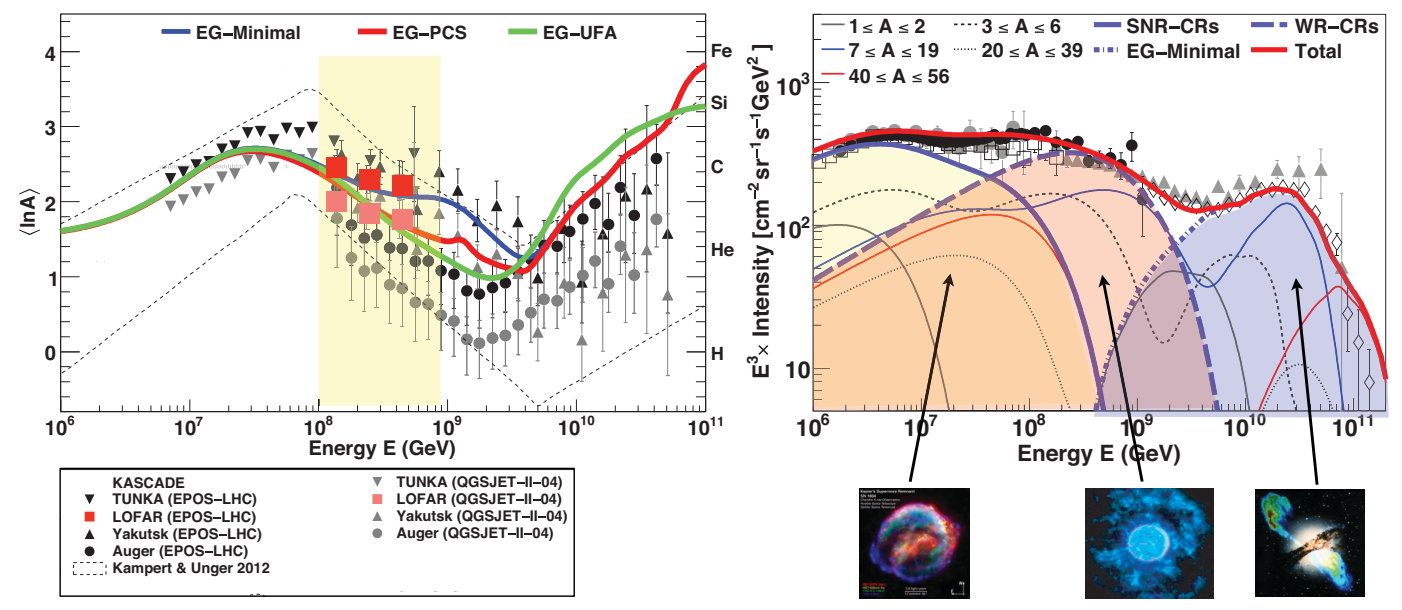

Figure 2. Left: Mean logarithmic mass of cosmic rays as a function of energy, for details, see [30]. Right: Threecomponent model of the origin of cosmic rays according to [30]: 'regular' supernovae, Wolf Rayet component, and an extra-galactic component.

\section{Particle type}

The good agreement between the measurements and the predictions of the CoREAS code is essential to identify the type of incoming cosmic ray. This is inferred from the (atmospheric) depth of the shower maximum $X_{\max }$, one of the standard measures to estimate $\ln A$. To measure $X_{\max }$ [26, 29] we analyse simultaneously measurements of the radio emission and the particle detectors. The arrival direction and energy of each cosmic ray are determined first. Then, simulations for primary protons and iron nuclei are conducted for each measured shower with its corresponding direction and energy. Due to the intrinsic shower fluctuations is it sufficient to simulate only protons and iron nuclei to cover the parameter space in $X_{\max }$. The predictions for the signals in the particle detectors and the radio antennas are compared on a statistical basis to the measured values. This method is used to determine $X_{\max }$ with an accuracy of better than $\sim 20 \mathrm{~g} / \mathrm{cm}^{2}$ with the dense LOFAR core, thus, reaching the state of the art - the uncertainty of the Pierre Auger Observatory fluorescence detector. The $X_{\max }$ values obtained are depicted as a function of energy in Fig. 1 together with other measurements. The latter apply different techniques, namely measuring Cherenkov and fluorescence light from the air showers. The figure illustrates the good agreement between the radio measurements and the established optical methods.

The measured values for the depth of the shower maximum $X_{\max }$ are converted to the mean logarithmic mass of cosmic rays

$$
\langle\ln A\rangle=\left(\frac{X_{\max }-X_{\max }^{\mathrm{p}}}{X_{\max }^{\mathrm{Fe}}-X_{\max }^{\mathrm{p}}}\right) \times \ln A_{\mathrm{Fe}} .
$$

This necessiates predictions for the depth of the shower maximum for impinging protons and iron nuclei, $X_{\max }^{\mathrm{p}}$ and $X_{\max }^{\mathrm{Fe}}$, respectively. These are illustrated as lines in Fig. 1: protons (green) and iron 
nuclei (red). The resulting mean mass is depicted in Fig. 2 (left) as a function of energy for the LOFAR results together with the world data set [30]. Two hadronic interaction models are used (EPOS-LHC and QGSJETII.04, dashed and solid lines in Fig. 1, respectively) to interpret the data. Both interaction models are tuned to LHC data but uncertainties remain, when extrapolating to the cosmic-ray parameter space.

\section{Origin of cosmic rays}

To understand the implications of the LOFAR measurements and the available world data set from direct and indirect measurements a model has been developed to consistently describe the observed energy spectrum and mass composition of cosmic rays with energies up to about $10^{18} \mathrm{eV}$ [30]. We assume that the bulk of Galactic cosmic rays is accelerated by strong Supernova remnant shock waves [31]. Our study shows that a single Galactic component with rigidity-dependent energy cut-offs in the individual spectra of different elements cannot explain the observed all-particle spectrum at energies exceeding $\sim 2 \cdot 10^{16} \mathrm{eV}$. Similar findings have already been obtained earlier [32]. We discuss two approaches for a second component of Galactic cosmic rays: re-acceleration at a Galactic wind termination shock and Supernova explosions of Wolf-Rayet stars. The latter scenario can explain almost all observed features in the all-particle spectrum and the mass composition of cosmic rays up to $\sim 10^{18} \mathrm{eV}$, when combined with a canonical extra-galactic spectrum as expected from strong radio galaxies or a source population with similar cosmological evolution. The resulting spectrum is shown in Fig. 2 (right). In this two-component Galactic cosmic-ray model, the 'knee' at $\sim 4 \cdot 10^{15} \mathrm{eV}$ and the 'second knee' at $\sim 4 \cdot 10^{17} \mathrm{eV}$ in the all-particle spectrum are due to the fall-offs of the first and second Galactic cosmic-ray components, respectively.

\section{Summary}

The radio detection of extensive air showers enables us to measure the properties of cosmic rays above energies exceeding $10^{17} \mathrm{eV}$ with high precision of $\sim 0.1^{\circ}-0.5^{\circ}$ for the arrival direction, $\sim 30 \%$ for the energy, and to better than $\sim 20 \mathrm{~g} / \mathrm{cm}^{2}$ for the depth of the shower maximum $X_{\max }$.

To illustrate the potential of the LOFAR radio measurements we developed a model to consistently describe the observed energy spectrum and mass composition of cosmic rays from $\mathrm{GeV}$ energies up to $10^{20} \mathrm{eV}$. We adopt a three component model: 'regular' cosmic rays being accelerated in Supernova remnants up to $\sim 10^{17} \mathrm{eV}$, a second Galactic component, dominating the all-particle flux between $\sim 10^{17}$ and $\sim 10^{18} \mathrm{eV}$ from cosmic rays being accelerated by exploding Wolf-Rayet stars, yielding a strong contribution of $\mathrm{He}$ and $\mathrm{CNO}$ elements, and, finally, an extra-galactic contribution at energies above $\sim 10^{18} \mathrm{eV}$.

\section{Acknowledgements}

JRH is grateful to the organizers of the RICAP conference for their kind invitation and the great hospitality.

\section{References}

[1] F. Aharonian et al., Astron. \& Astroph. 449, 223 (2006)

[2] H. Völk, E. Berezhko, Astron. \& Astroph. 451, 981 (2006)

[3] J. Blümer, R. Engel, J.R. Hörandel, Prog. Part. Nucl. Phys. 63, 293 (2009) 
[4] M. Nagano, A. Watson, Rev. Mod. Phys. 72, 689 (2000)

[5] J.R. Hörandel, Nucl. Instr. \& Meth. A 588, 181 (2008)

[6] T. Huege, Phys. Rept. 620, 1 (2016), arXiv: 1601.07426

[7] F.G. Schröder, Prog. Part. Nucl. Phys. 93, 1 (2017), arXiv: 1607.08781

[8] M. van Haarlem et al., Astron. \& Astrophys. 556, A2 (2013), arXiv: 1305 . 3550

[9] P. Schellart et al., Astron. \& Astrophys. 560, A98 (2013), arXiv: 1311.1399

[10] A. Nelles et al., JINST 10, P11005 (2015), arXiv: 1507.08932

[11] S. Thoudam et al., Nucl.Instrum.Meth. A767, 339 (2014), arXiv: 1408 . 4469

[12] S. Thoudam et al., Astropart. Phys. 73, 34 (2016), arXiv: 1506.09134

[13] T. Huege, M. Ludwig, C.W. James, AIP Conf. Proc. 1535, 128 (2013), arXiv: 1301.2132

[14] J.R. Hörandel et al., Proceedings of the 33rd International Cosmic Ray Conference, Rio de Janeiro \# 865 (2013)

[15] J.R. Hörandel et al., Proceedings of the 34th International Cosmic Ray Conference, Den Haag PoS(ICRC2015)033 (2015)

[16] J.R. Hörandel, JPS Conf. Proc. 9, 010004 (2016), arXiv : 1509.04960

[17] A. Nelles et al., Astropart.Phys. 60, 13 (2015), arXiv: 1402.2872

[18] A. Nelles et al., JCAP 1505, 018 (2015), arXiv: 1411.7868

[19] A. Corstanje et al., Astropart.Phys. 61, 22 (2015), arXiv: 1404 . 3907

[20] P. Schellart et al., JCAP 1410, 014 (2014), arXiv: 1406.1355

[21] O. Scholten et al., Phys. Rev. D94, 103010 (2016), arXiv: 1611.00758

[22] A. Nelles et al., Astropart.Phys. 65, 11 (2014), arXiv: 1411.6865

[23] P. Schellart et al., Phys.Rev.Lett. 114, 165001 (2015), arXiv: 1504.05742

[24] T.N.G. Trinh et al., Phys. Rev. D93, 023003 (2016), arXiv: 1511.03045

[25] W. Apel et al., JCAP 1409, 025 (2014), arXiv: 1404.3283

[26] S. Buitinket al., Phys.Rev. D90, 082003 (2014), arXiv: 1408.7001

[27] A. Aab et al., Phys. Rev. D93, 122005 (2016), arXiv: 1508.04267

[28] A. Aab et al., Phys. Rev. Lett. 116, 241101 (2016), arXiv: 1605.02564

[29] S. Buitink et al., Nature 531, 70 (2016), arXiv : 1603.01594

[30] S. Thoudam, J.P. Rachen, A. van Vliet, A. Achterberg, S. Buitink, H. Falcke, J.R. Hörandel, Astron. \& Astrophys. 595, 33 (2016), arXiv: 1605.03111

[31] S. Thoudam, J.R. Hörandel, Astron. \& Astrophys. 567, A33 (2014), arXiv : 1404 . 3630

[32] A.M. Hillas, J. Phys. G31, R95 (2005) 